\title{
Study on the characteristics of fault in PuTaohua oil layer of GuLong Oilfield
}

\author{
Haifeng Chen, Xixin Wang, Qingyou Yue, Yuechao Teng, Haiqiang Hou, \\ Liang Wang \\ 1 Northeast Petroleum University unconventional oil-gas accumulation-development provincial jointly \\ key laboratory, Daqing, Heilongjiang, China, 163318
}

\begin{abstract}
In order to study the relationship between the faults in PuTaohua oil layer of GuLong Oilfield and the distribution of oil and water, the $\mathrm{T}_{11}$ fault series were studied which are closely related to target stratum, analysied of geometry and kinematics characteristics of faults systematically, stduied the influence on the distribution of oil and water that faults as oil and gas migration path and fault sealing, that layed the foundation for oil and gas migration and accumulation of this study area.
\end{abstract}

Keywords: - fracture system geometry characteristics kinematics characteristics closure

\section{INTRODUCTION}

Fracture is the most common sedimentary basin tectonic deformation styles ${ }^{[1,2]}$, research on the relationship between the fracture and the reservoir has been a focus of research in petroleum geology ${ }^{[3]}$. Gu Long oilfield located in convert site between GaoXi nose structure and LongNan nose structure, it is saddle of a hill of Talaha syncline and Changjiaweizi syncline. It east to the Gaotaizi oilfield, and south to Puxi oilfield and Gaoxi oilfield, and west to Talaha oilfield,and norst to Longhupao oilfield and Longnan oilfield ${ }^{[4 \sim 6]}$. It`s structural trend is SN-NNE, fracture and local structures are well developed. Combining seismic data and drilling data, and on the basis of detailed interpretation of the fault, the fracture system features were fine anatomized.

\section{FRACTURE CHARACTERISTICS ANALYSIS}

In order to analyze oil and gas migration and accumulation mode, forming rules, main controlling factors, we must have deep knowledge and understanding of the characteristics of fault system. In this study, $\mathrm{T}_{11}$ fault is relatively more developed, the characteristics of fracture is obvious, So we started studying $T_{11}$ fault rupture characteristics from geometry, kinematics characteristic fracture, analysised of the closed nature of the zone.

\subsection{Fracture geometry features}

1.1.1 The planar characteristics

Strike, dip direction and dip angle is the three elementsof fault occurrence, but also the basis for the analysis of fault geometry. Fault geometry distribution of Putaohua oil layer of GuLong Oilfield e.g. Fig .1 


\begin{tabular}{|c|c|c|c|}
\hline trend the rose diagram & extended length $(\mathrm{m})$ & fault distance $(\mathrm{m})$ & $\begin{array}{c}\text { the range of dip } \\
\text { angle }\end{array}$ \\
\hline Fig.1 & & \\
\hline
\end{tabular}

In the statistics of 439 faults in this area, the fault strike is mainly in the range of NWW-NW, NW and NNE trending faults are less, the range of dip angle is $78^{\circ} \sim 89^{\circ}$. The fault strike and the structural contour are approximately vertical, this This shows that the area is affected obviously by the northeast to the compressional stress, lead to the formation of today's most high angle normal faults.

\subsubsection{Fault density}

In the study area, the total number of T11 reflectionlayer fracture is 439 , research area is $1067 \mathrm{~km} 2$, average fracture density is $0.41 / \mathrm{km} 2$.

\subsubsection{Fault dimention}

The fault dimention refers to the fault plane extending length and displacementand the relationship between the two ${ }^{[7,8]}$, analysis of fault dimention helps to study the formation mechanism and activity patterns. In general the tectonic faults have the characteristics ofpower-law distribution, performance for the scale invariant characteristics, that is self-similar, but there are also non power law distribution characteristics of fault, which are called scale-bound fault system, this type of fault may be non tectonic origin. e.g. Fig.2

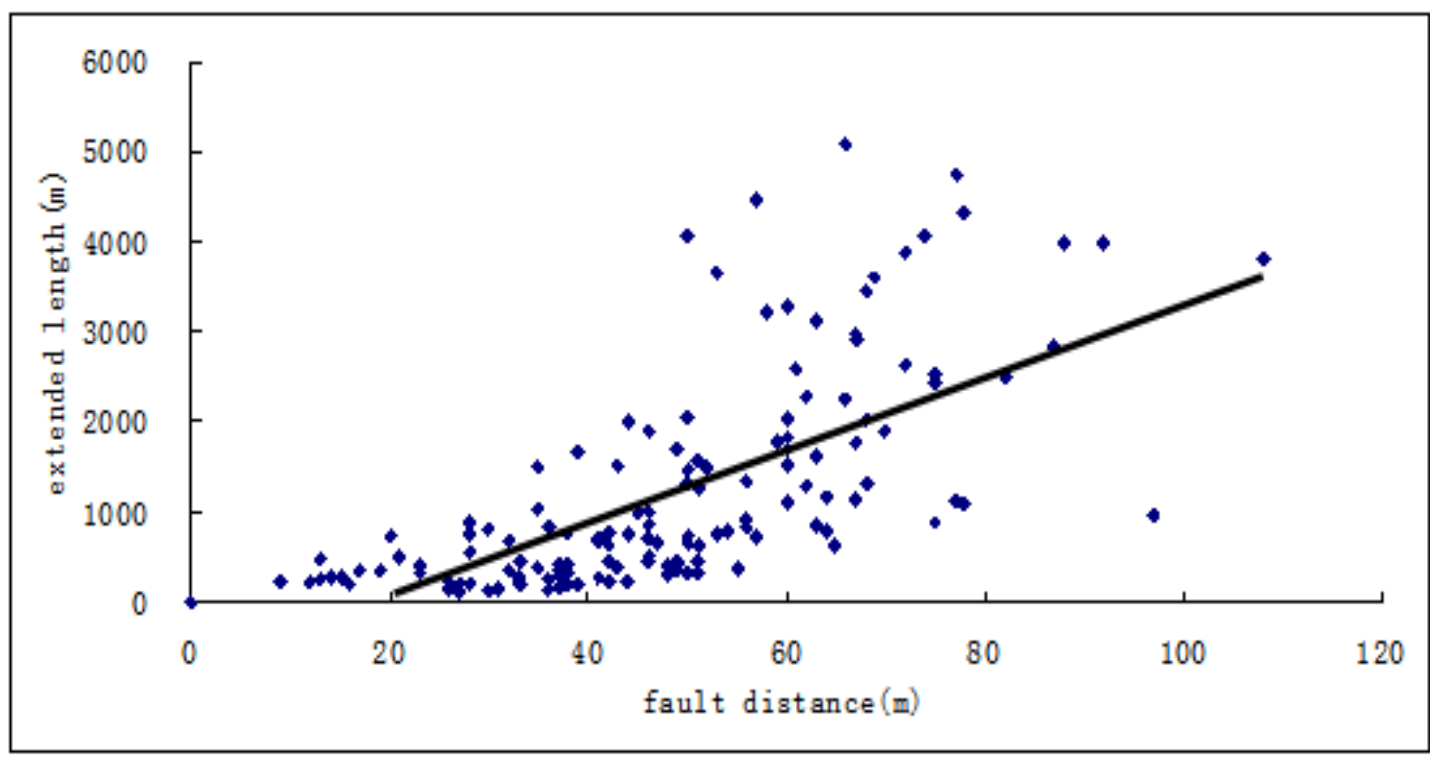

Fig 2

As can be seen from the diagram, fault extending length and fault distance has a linear relationship, that is tectonic origin. 


\subsubsection{Fault combination mode}

In the tectonic evolution of the basin, although different scholars have different basin mechanism based on analysis and discussion of different, but basically agree with the fault depression,depression and reversal of three large tectonic evolution stage, the superimposed basin mode is two structure of "down fault up concave". Accordingly divided into three structural layers: rift structure layer, depression structure layer and inversion structure layer. In this region, the tectonic evolution can be divided into two fault lines: the fault depression layer fault systemand depression layer fault system. e.g. Fig.3.

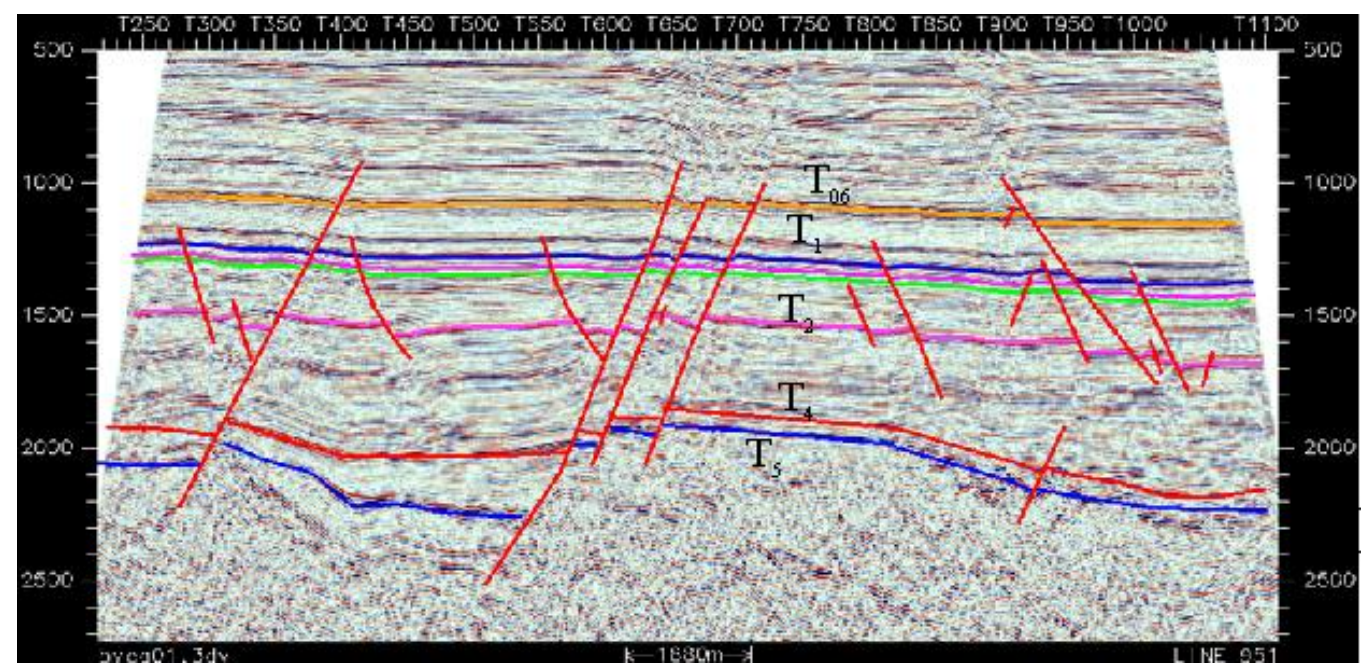

Fig.3. fault combination

Fault system in the upper basin mainly has threemodes: "V" type, "Y and anti y font", "Domino effect"; Patternof lowerrifting fault depressioncontrolling large-scale backbone fault mainlyhas:

the ladder, shovel,Horst and graben; The two upper and lower fault system in sectioncombined into a "flower like" fault pattern ,"Y and Y" font fault combination mode.

\section{THE KINEMATICS CHARACTERISTICS OF THE FAULT}

The fracture kinematics is part of tectonickinematics, which mainly study eolutional history and activity of fault. In the present study, mainly from the fault period, active period, breaking away from aspects of characteristicswere studied, in order to investigate the kinematics characteristics of the fault in the area.

Fault stage classification

On the basis of the formation and evolution of fault time,fault system can be divided into 4 types (long, early,middle and late), 9 types(according to break through the layers). The middle fracture mainly control Putaohua reservoir. According to the main horizons off wear and oil and gas accumulation contribution is divided into nine categories, respectively: $T_{5}-T_{06}, T_{5}-T_{1}, T_{4}-T_{11}, T_{2}-T_{1}, T_{2}-T_{11}$, $\mathrm{T}_{2}-\mathrm{T}_{06}, \mathrm{~T}_{1}-\mathrm{T}_{1}, \mathrm{~T}_{1}-\mathrm{T}_{0} 6$, only cut across $\mathrm{T}_{11}$, these faults constitute the $\mathrm{T}_{11}$ fault system of the research area. e.g. Fig.4. 


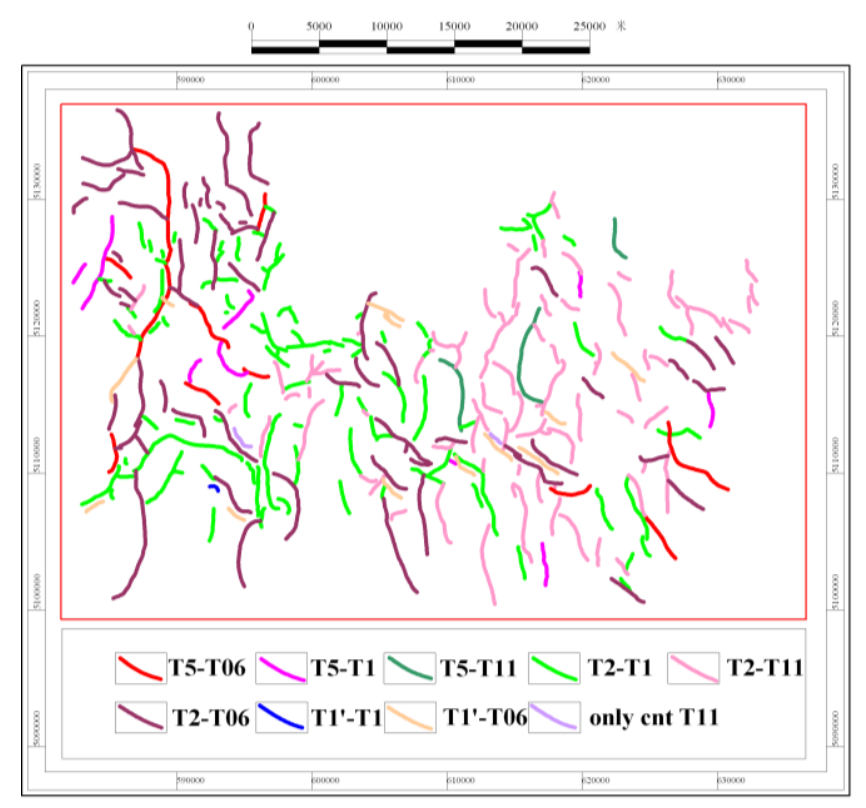

Fig.4. fault periods

According to the evaluation of hydrocarbon source rock in the study area shows, Gulong oilfield oil and gas sources is the vertical migration, lower oil generatingcapacity is relatively strong. Oil and gas of Putaohua reservoir comed mainly from the lower member 1 of Qingshankou source rock, while communication oil source and destination layerthrough long-term fault each other, figure T5-T06, T5-T1, T4-T11 type of fault is the fault.

\section{IVANALYSIS OF FAULT SEALING}

By using the fault distance and the faulted stratamudstone calculating any docking area SGR cumulative thickness size, SGR is shale gouge ratio in the fault zone ${ }^{[9,10]}$.

$$
S G R=\frac{\sum(V s h \bullet \Delta Z)}{D} \times 100 \%
$$

SGR of above formulais shale gouge ratio in the fault zone; Vsh is shale percentage content, $\%$; $\Delta Z_{\text {is stratum }}$ thickness of the fault distance range, $\mathrm{m}$; D is fault distance, $\mathrm{m}$;

Combined with the local practice, use the following SGR standard table.

\begin{tabular}{|c|c|c|c|c|}
\hline Sealing evaluation & bad & medium & better & best \\
\hline SGR $(\%)$ & $<30$ & $30 \sim 50$ & $50 \sim 70$ & $>70$ \\
\hline
\end{tabular}




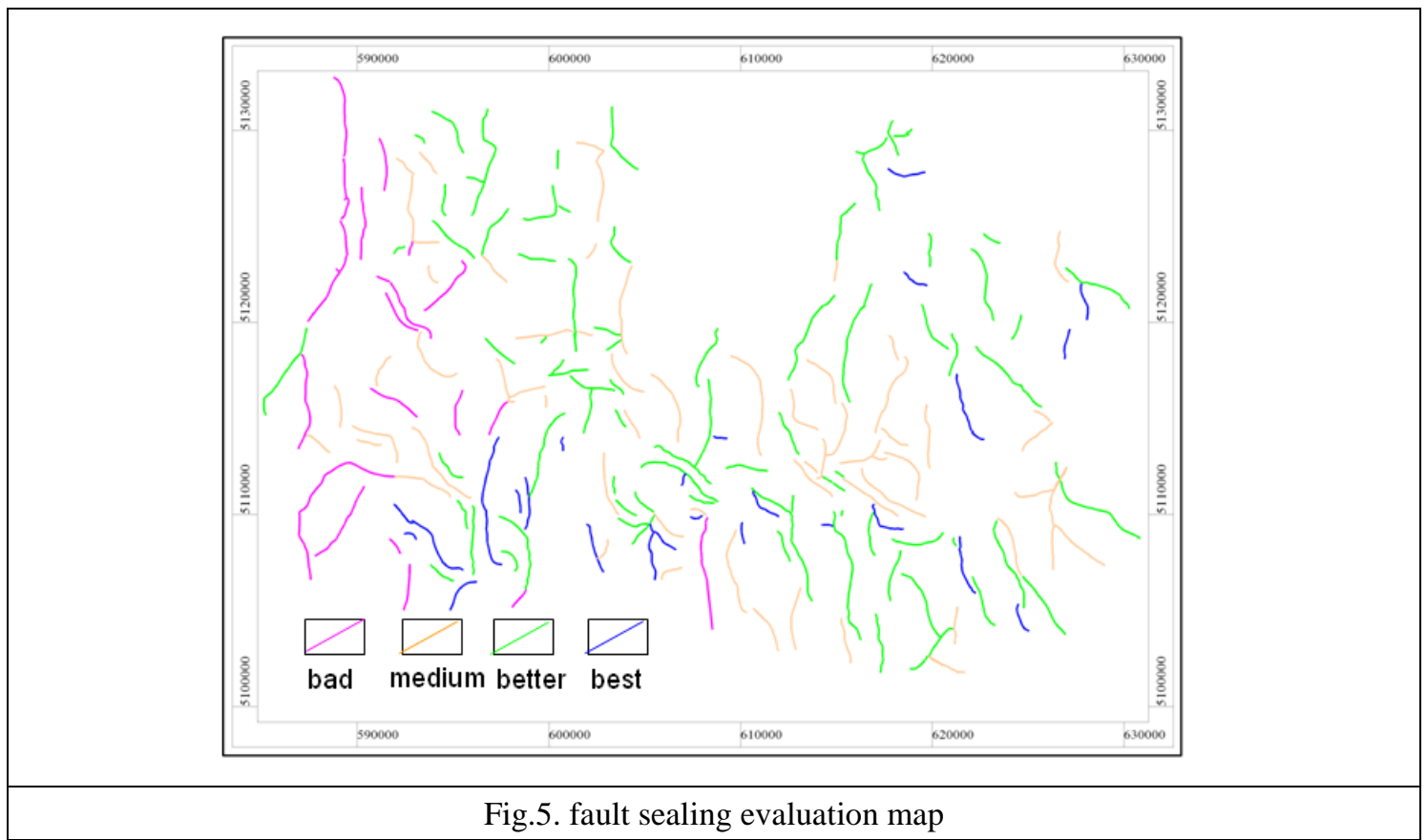

The main types of the lateral sealing of faults are the medium, the better, and the bad concentrated in the gentle slope zone in western area.

\section{CONCLUSION}

A, The faults of Putaohua reservoir of Gulong oilfield, fault strike is mainly in the range of NWW-NW.

B, The T5-T06, T5-T1, T4-T11 faults is oil-gas communication channel in the research area.

C, Oil water distribution of the study area is not only controlled by faults ,but also by the lithology, reservoir parameter, sedimentary rhythm,pore throat structure.

\section{REFERENCES}

[1] Fu Xiaofei, Li Wenlong, Lu Yan Fang, He Xiangyang,Liu Zhe. Fault lateral sealing and it`s control to the distribution of oil and water. Geological review. 2011,3(57):386 397.

[2] Sun Yonghe,Han Yuping,Feng Zhipeng,Fu Xiaofei,Liu Zhe. The fracture system and its control effect on hydrocarbon migration and accumulation in Beier sunken of Hailaer basin. Geological review,2011,1(57):89 100.

[3] Chen Fangwen,Lu Shuangfang,Shi Meijuan. The faylt features and its control effect on oil-gas. Journal of Central South University(NATURAL SCIENCE EDITION) (43):249 257.

[4] Liu Gang. Oil and gas enrichment conditions of the G88 development zones in Putaohua oil layer of GuLong Oilfield.

[5] Pang Yanming,Liao Yuanhui,Zhou Yongbing,Wang Xiujuan,Wang Xuefeng. The formation of the main controlling factors anddistribution of oil-water layer reservoir of GuLong Oilfield. The fifth session of the International Conference on oil and gas accumulation mechanism and evaluation of oil and gas resources set, 2009,10.

[6] Chen Lei, Ma Shizhong, Fu Xian article, sun rain, Fan Shuhao. The main controlling factors of oil and gas enrichment in Putaohua oil layer of Gaotaizi Oilfield of Gulong depression. Inner Mongolia Petrochemical 2010 (7): 52 54.

[7] Liu Qidong,Li Chuhua,Lu Lixia. Study on the fault sealing properties of Gaoyou depression. Journal of natural gas chemistry (Journal of Jianghan Petroleum Institute), 2010.4 (32): 58 61. 
[8] Lv Yanfang,sand Xuan,Fu Xiaofei,Fu guang. The fault vertical sealing evaluation methodof quantitative and its application. Acta petrolei Sinica, 2007,9 (28): 35 39.

[9] Yielding G, Freeman B, Needham D T. Quantitative Fault Seal Prediction[J]. AAPG Bulletin, 1998, 81 (6): 897 917.

[10] Lv Yanfang, Fu Guang, Gao Da Ling, et al. Oil and gas reservoir capping research. [M]. Beijing: petroleum industry press, $1996,55-123$. 